\title{
MIGRACIÓN INDÍGENA, PROCESOS DE TERRITORIALIZACIÓN Y ANÁLISIS DE REDES SOCIALES
}

\author{
Carolina Andrea Maidana*
}

El presente trabajo discute los alcances y las limitaciones del Análisis de Redes Sociales en el estudio de las migraciones indígenas y los consecuentes procesos de territorialización, a partir de los datos obtenidos en un estudio sobre la conformación de los denominados "barrios toba" en las periferias de grandes ciudades. Para ello se describe y analiza la red social que vincula - a través del parentesco - a los habitantes de uno de estos barrios, destacando la relación existente entre la centralidad de cada individuo en la arquitectura de la red y su contribución a explicar tanto el sentido de la migración, como el posterior asentamiento en una zona determinada.

Palabras clave: indígenas, redes sociales, parentesco, migración, procesos de territorialización.

\section{Introducción}

El presente trabajo es resultado del análisis, desde una perspectiva de redes y a luz de las nuevas tecnologías informatizadas, de datos obtenidos en una investigación etnográfica realizada entre los años 2006 - 2011,1 junto a migrantes indígenas qom (toba) ${ }^{2}$. En la misma analizamos la existencia de múltiples nucleamientos ${ }^{3}$ constituidos en el camino de la

\footnotetext{
* Facultad de Ciencias Naturales y Museo, Universidad Nacional de la Plata. Buenos Aires/Argentina.

${ }^{1}$ MAIDANA, Carolina Andrea. Migrantes toba (qom). Procesos de territorialización y construcción de identidades.

2 "Qom" es la forma en que se autodenomina el pueblo indígena conocido como toba; pueblo de cazadores, pescadores y recolectores nómades habitaba, antes de la conquista y colonización, la región geográfica de América del Sur denominada Gran Chaco.

${ }^{3}$ Utilizamos este término para referirnos a los barrios / comunidades / asentamientos de población qom migrante constituidos en torno a grandes ciudades (cf. TAMAGNO, Liliana. NAM QOM HUETA'A NA DOQSHI LMA'. Los tobas en la casa del hombre blanco. Identidad, memoria y utopía) con el objetivo de dar lugar a la comprensión de los desplazamientos y las formas en las cuales los migrantes qom se concentran en el espacio.
} 
migración, vinculando la distribución de los mismos con el parentesco, lo cual implicó el análisis de las redes de relaciones parentales que unen a los migrantes entre sí, y con los lugares de origen. Para ello efectuamos observación participante/participación objetivante, trabajamos con relatos de experiencias migratorias e historias de vida, realizamos encuestas, entrevistas abiertas y semi-estructuradas, relevamos genealogías y diagramas de parentesco [Imagen I], recurrimos al registro fotográfico y utilizamos un software especializado (UCINET-NETDRAW) ${ }^{4}$ para visualizar y analizar, las redes sociales centrándonos en el nucleamiento más antiguo de la ciudad de La Plata ${ }^{5}$. Fue así que observamos y participamos de una serie de acontecimientos, que más tarde analizaríamos, en tanto indicadores de la dinámica sociocultural que involucra a este nucleamiento en particular, y a toda una constelación de organizaciones y familias indígenas que residen en diferentes espacios, tanto en los lugares de origen como en los de migración. Lugares que además de visitar personalmente, pudimos conocer a través de los relatos de nuestros interlocutores que nos fueron llevando y trayendo continuamente desde la espesura del monte a la densidad de la ciudad.

\section{IMAGEN I \\ Diagrama realizado por una joven indígena qom en el contexto del LIAS Ciudad de La Plata año 2007}

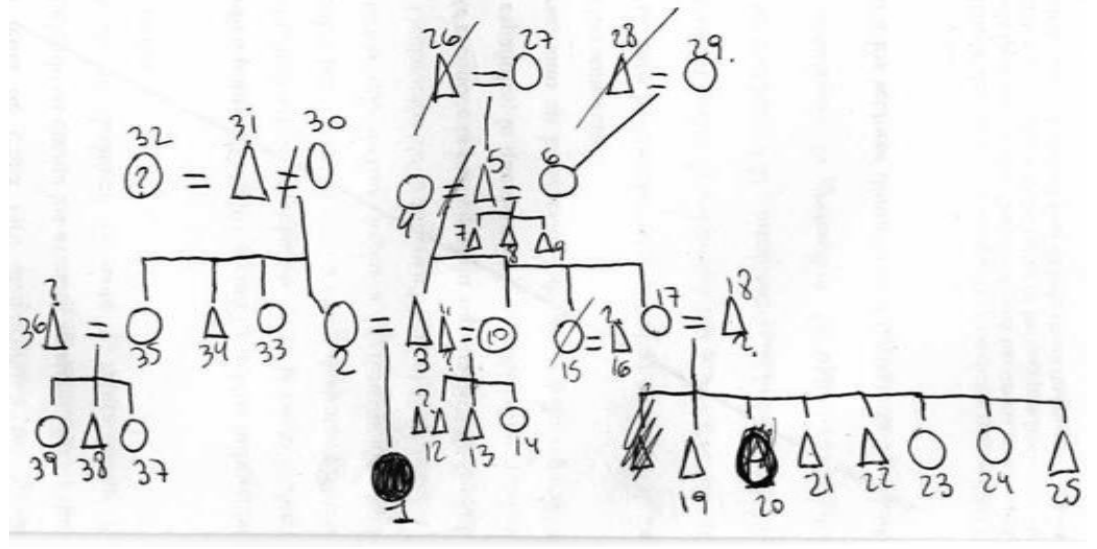

Las migraciones en general e indígenas en particular están ligadas a los procesos sociopolíticos y económicos de la estructura social en la cual se desarrollan. De allí la imposibilidad de comprenderlos de forma aislada,

\footnotetext{
${ }^{4}$ BORGATTI, Stephen P.; EVERETT, Martin G; FREEMAN, Lin C. Ucinet for Windows: Software for Social Network Analysis. Harvard, MA: Analytic Technologies, 2002.

${ }^{5}$ Capital de la provincia de Buenos Aires, ubicada a $56 \mathrm{Km}$. al sudeste de la ciudad homónima.
} 
disociados de la dinámica de la estructura social en la cual se insertan y la necesidad de considerarlos como fenómenos sociales íntimamente relacionados con la situación de contacto interétnico ${ }^{6}$. Para el caso de los pueblos indígenas del Gran $\mathrm{Chaco}^{7}$ el descubrimiento, la conquista y la colonización de estas tierras, al igual que las posteriores transformaciones generadas por la gestación y consolidación del Estado-Nación argentino, junto a la expansión de los emprendimientos productivos y la evangelización, entrañaron profundos cambios en sus condiciones materiales de existencia. Estos procesos, guiados por una lógica de destierro, los expulsaron, confinaron y despojaron de las tierras que ocupaban. Si bien los contactos iníciales con los europeos se establecieron a principios del siglo XVI, desde entonces y hasta el último cuarto del siglo XIX los indígenas del Gran Chaco lograron mantener la frontera con los blancos, con quienes establecieron contactos intermitentes y restringidos a aspectos militares, acuerdos políticos y/o incursiones misionales. Ello se debió a que la economía colonial, basada en la extracción y en la acumulación, no necesitaba de una expansión colonizadora ${ }^{8}$. Pero una vez consolidado el Estado-Nación argentino, se inició un período de organización en el cual sucesivas expediciones militares fueron emprendidas para delimitar el espacio terrestre sobre el cual se afirmaría el control estatal. A partir de la ocupación del Gran Chaco por el Ejército Nacional, en la década de 1880, esta región se abriría a la penetración de una economía política capitalista signada por el establecimiento de ingenios azucareros, la implantación de explotaciones forestales y el desarrollo de una agricultura intensiva, situación que fue acompañada por una fuerte presencia de la evangelización protestante.

El derrocamiento del gobierno popular del Gral. Juan Domingo Perón en 1955, marcaría un momento significativo en la neutralización/ transformación del modelo de redistribución acorde a lo que se ha denominado "Estado de Bienestar", para dar lugar a un modelo fundado en la libertad de mercado que condujo a un nuevo reordenamiento en las ofertas laborales. Cada una de estas etapas de contacto entre indígenas y blancos por la que atravesó la región chaqueña produjo fuertes desplazamientos

6 FÍGOLI, Leonardo; Dimitri, FAZITO. Redes sociales en una investigación de migración indígena: el caso de Manaus.

7 Región que se extiende entre la precordillera andina -al oeste-, los ríos Paraguay y Paraná -al este-, la Meseta del Mato Grosso -al norte- y la cuenca del río Salado -al sur-, comprendiendo parte de las actuales Argentina, Paraguay y Bolivia. El Chaco Argentino abarca en la actualidad, y de acuerdo a las divisiones políticas, siete provincias: Formosa, Chaco, este de Salta, este de Tucumán, noreste de Santiago del Estero, noreste de Córdoba y norte de Santa Fe.

8 BARTOLOMÉ, Miguel. Los pobladores del desierto. Genocidio, etnocidio y etnogénesis en la Argentina. 
de indígenas a consecuencia de la violenta penetración económica, estatal y religiosa en sus territorios ancestrales. La reducción de los espacios efectivamente apropiados y las posibilidades de subsistencia, los condujo no sólo a las periferias de las ciudades y poblados de la región, sino también a las periferias de grandes centros urbanos como Rosario ${ }^{9}$, Buenos Aires ${ }^{10}$ y La Plata donde constituyeron los denominados "barrios toba" 11 .

El "barrio toba" donde realizamos trabajo de campo de forma sostenida en el tiempo comprende 36 familias nucleadas en torno a una Asociación Civil. Estas familias se asientan en el contexto mayor del denominado "barrio Malvinas", unidad administrativa reconocida por la municipalidad de la ciudad de La Plata. La historia de este nucleamiento indígena se remonta al año 1991, en que se inició un programa de radicación de familias dependiente de la Dirección de Ordenamiento Urbano de la Provincia de Buenos Aires, en el marco del Programa "PRO-TIERRA" del gobierno provincial; programa que apuntaba a atender el déficit habitacional en la región al contemplar la venta de terrenos con provisión de agua corriente, desagües cloacales y pluviales, alumbrado público, electricidad y apertura de calles así como también la autoconstrucción de viviendas.

Desde el enfoque propuesto intentamos reconstruir la red utilizada por los migrantes para insertarse en el lugar de llegada, organizarse y acceder a la vivienda. La relación entre migración y reconstrucción territorial es abordada a partir de los conceptos de red y cadena, conceptos que habilitan el análisis de la constitución y organización de los denominados "barrios tobas" a partir de la transposición, reproducción y recreación de redes sociales en nuevos contextos espaciales. En este trabajo en particular mostramos, a partir del análisis de la red social que vincula -a través del parentesco ${ }^{12}$ - a los habitantes del citado nucleamiento, cómo la aplicación de las nuevas tecnologías informatizadas para el análisis de redes (ARS) nos permitió reforzar los argumentos obtenidos durante el trabajo de campo.

\footnotetext{
${ }^{9}$ Es una de las ciudades más populosas del país, situada sobre la margen occidental del río Paraná, en la provincia de Santa Fe.

${ }^{10}$ Ciudad capital de la República Argentina, situada en la provincia homónima, sobre la orilla occidental del Río de la Plata.

11 Porciones de ciudad reconocidas y caracterizadas por la cohesión/identificación étnica de los conjuntos de familias que los habitan, sin necesidad de que éstos conjuntos sean definidos desde el punto de vista administrativo.

12 Nos referimos aquí a la red privilegiada de relaciones prácticas que comprende no solamente al conjunto de relaciones genealógicas, sino también a aquellas relaciones no genealógicas que pueden son movilizadas por las necesidades ordinarias de la existencia, es decir al "parentesco práctico" (BOURDIEU, Pierre. El sentido práctico).
} 


\section{Redes, cadenas y trayectorias socioespaciales: nucleamientos indígenas en grandes ciudades}

Los tres tipos de funciones cumplidas por las redes migratorias ${ }^{13}$ : de adaptación (acomodamiento a las condiciones de llegada e integración en las instituciones principales de la sociedad de destino), de selección (determinación de qué individuos migran) y de canalización (transmisión de recursos necesarios para el desplazamiento) se corresponden con las tres etapas de los procesos migratorios señaladas por Eisenstadt ${ }^{14}$ : 1) las motivaciones para migrar (circunstancias del lugar de origen o "factores de expulsión", posibilidades y disponibilidades en el lugar de destino o "factores de atracción"), 2) la estructura social del proceso migratorio (características del traslado), y 3) la incorporación de los migrantes a un nuevo marco social y cultural (integración recíproca entre migrantes y sociedad receptora).

Sobre el primer aspecto de la migración, en América Latina en general las denominadas "migraciones internas", de carácter rural-urbano, se incrementaron exponencialmente durante la segunda mitad del siglo XX, asociadas a la expansión industrial y a la urbanización, a la demanda de mano de obra en las ciudades y a la expulsión de población campesinoindígena que se vio empobrecida y desempleada ante el avance de la frontera agrícola y debido a que su labor fue suplida por moderna maquinaria. En Argentina, en particular, los desplazamientos internos comenzaron alrededor de 1930, coincidiendo con la crisis económica que determinó la merma de las importaciones y favoreció el desarrollo industrial al forzar la sustitución de las mismas. El rápido crecimiento industrial, emplazado a orillas del Río de la Plata y el Río Paraná generó ofertas de trabajo en Buenos Aires y Rosario que motivaron, a mediados del siglo XX, una fuerte migración rural-urbana hacia estas ciudades. A mediados del siglo XX cuando la industria iniciaba su retracción, el proceso migratorio continuó, y fue en esta época cuando se sucedieron los primeros flujos significativos de familias indígenas qom a los grandes centros urbanos antes mencionados ${ }^{15}$. Es por ello que entendemos a las migraciones como un fenómeno recurrente y constante -aunque se densifique o flexibilice según períodos- históricamente relacionado con la expansión colonial y por lo tanto inescindible de los violentos y traumáticos

\footnotetext{
${ }^{13}$ Gurak y Caces 1998, apud BASSARSKY, Lina. Enfoque de redes sociales en las migraciones de América latina hacia Francia.

${ }^{14}$ EISENSTADT, Shmuel N. The absorption of Inmigrants.

${ }^{15}$ Ver TAMAGNO, op. cit.; BIGOT, Margot; RODRÍGUEZ, Graciela; VÁSQUEZ, Héctor. Asentamientos Toba-Qom en la ciudad de Rosario. Procesos étnicos identitarios; ARIAS, Nora Julia. Vueltas y revueltas (estratégicas) de los Toba rosarinos - de Resistencia a Rosario; y WRIGHT, Pablo. Histories of Buenos Aires, entre otros.
} 
procesos de destierro y expropiación a los que se vieron y aún se ven sometidos campesinos e indígenas ${ }^{16}$.

Sobre el segundo aspecto, consideramos a las denominadas "cadenas migratorias" como factor clave en la decisión de migrar y en la selección del lugar hacia el cual dirigirse y en el cual asentarse. De allí que analicemos las redes de relaciones parentales como uno de los factores significativos para explicar tanto los procesos de migración como la reconstrucción territorial e identitaria de los migrantes qom. Pues, en los testimonios recabados aparece siempre la referencia a algún pariente que, habiendo migrado antes, informaba sobre las posibilidades/disponibilidades que ofrecía el lugar donde se había instalado; algo que, desde una perspectiva territorial, se manifiesta en la concentración y/o localización puntual de la población qom migrante $^{17}$. No sólo la noción de cadena sino también la de red se muestran esenciales para comprender el modo en que los procesos migratorios y de reconstrucción territorial e identitaria conforman y caracterizan la dinámica sociocultural del Pueblo Qom. Estas nociones permiten hacer visible la forma en que el parentesco se activa con la migración, permitiendo la circulación de ciertos recursos materiales y simbólicos necesarios e imprescindibles tanto para el traslado como para etapas posteriores de la experiencia migratoria, la instalación y organización en el lugar de destino. Las redes de relaciones que enlazan las áreas de origen y destino y éstas últimas entre sí son multidireccionales, algo que frecuentemente se desdibuja en los análisis migratorios al primar las perspectivas de la emigración o inmigración. Es por ello que sumamos a estas conceptualizaciones la utilización de la categoría de migrante, para referenciar la doble condición de emigrantes/inmigrantes de quienes habitan hoy la ciudad y protagonizan movimientos poblacionales constantes que vinculan/enlazan a los nucleamientos urbanos entre sí y con los lugares de origen (visitas, retornos temporarios, reemigraciones y/o retornos diferidos a los lugares de procedencia).

Los tradicionales modelos explicativos empleados para el análisis de los procesos migratorios se basan en la teoría del pull and push, que permite dar cuenta de las condiciones estructurales que actúan como factores de atracción y expulsión de población en ciertas localidades y/o regiones espacialmente delimitadas. Este marco analítico que fue utilizado por primera vez -aunque en forma implícita ${ }^{18}$ - por Ernest George Ravenstein ${ }^{19}$, permite

\footnotetext{
${ }_{16}$ MAIDANA, Carolina Andrea. Volver a la tierra. Parentesco, redimensionalización territorial y reconstrucción identitaria.

17 Ibidem.

18 ARANGO, Joaquín. Las 'leyes de las migraciones' de E. Ravenstein, cien años después.

${ }^{19}$ RAVENSTEIN, Ernest George. The Laws of Migration, 1885; IDEM. The Laws of Migration, 1889.
} 
explicar la migración rural-urbana en la época moderna y cuando ésta se presenta a escala masiva, al considerar los mecanismos de oferta y demanda de mano de obra y la industrialización. Sin embargo, esta relación causal, sólo esclarece parcialmente el fenómeno, ya que no permite dar cuenta de la selectividad de los migrantes, de las diversas formas de desplazamiento, ni de las variaciones ocurridas en distintos períodos históricos y en diferentes lugares ${ }^{20}$.

A los fines de superar las explicaciones unifactoriales de la migración, a partir de la década del 1950, se incorporaron las nociones red y cadena ${ }^{21}$ para el estudio de los movimientos poblacionales. Con ellas se complementó la mirada que observaba el contexto económico, político y socio histórico en el que las migraciones acontecían, con la comprensión de los migrantes en tanto sujetos activos y capaces de tomar -tras la consideración de numerosas alternativas- múltiples y diversas decisiones. Se destacó así la multivocalidad del fenómeno buscando superar cualquier tipo de interpretación mecánica del mismo. La aplicación de estos instrumentos metodológicos/herramientas analíticas implicó considerar aspectos relegados en los tradicionales modelos explicativos de la migración: la familia (expresada a través de los vínculos de parentesco); la dimensión local (materializada en relaciones de amistad y paisanaje); la interconexión que se produce entre las áreas de origen y destino $^{22}$; y los migrantes de diferentes períodos y los migrantes potenciales ${ }^{23}$. Es en este sentido que destacamos la utilidad no sólo de estos conceptos sino del análisis de redes a partir de la aplicación de softwares especializados, aunque su utilización sea poco frecuente dada la complejidad de los mismos y el tipo de información requerida, no ya de carácter atributivo sino relacional.

Entre los migrantes qom asentados en el nucleamiento de referencia la división de la tierra - a la que conduce el crecimiento vegetativo en los lugares de origen - y la falta de fuentes de trabajo - producto de los procesos de privatización y mercantilización de la tierra y de la industrialización son enunciadas recurrentemente como las causas de la migración; al mismo tiempo el alambrado y los carteles de "prohibido cazar" - expresiones del

\footnotetext{
${ }^{20}$ ARIZPE, Lourdes. Migración indígena, problemas analíticos; IDEM. Migración, etnicismo y cambio económico (un estudio sobre migrantes campesinos a la ciudad de México).

${ }^{21}$ MC DONALD, Leatrice; MC DONALD, John Stuart. Chain migration, ethnic neighborhood Formation and social networks.

${ }^{22}$ En Argentina Margullis fue pionero en el estudio de los procesos migratorios internos desde una perspectiva de totalidad, analizando no sólo los migrantes en la sociedad de origen y de destino sino también la inserción de este proceso dentro de la dinámica socioeconómica y cultural de la comunidad local, y sobre todo su relación con la estructura social global (MARGULLIS, Mario. Migración y marginalidad en la sociedad argentina, p. 12).

${ }^{23}$ RECAÑO VALVERDE, Joaquín. El papel de las redes en los procesos de migración interna.
} 
continuo avance de las fronteras agrícolas - son, según los testimonios, signos de estos procesos. Las configuraciones sociales y espaciales que han adoptado los qom en el camino de la migración dan cuenta de estos procesos pero, fundamentalmente, sus presencias citadinas, dan cuenta de las redes de relaciones que vehiculizan la migración, el acceso al suelo y la organización urbana. Cuando le preguntábamos a la gente qom de La Plata por la presencia de parientes en otros nucleamientos de Buenos Aires eran notables las unánimes respuestas afirmativas. Lo mismo sucedió en las visitas a los nucleamientos de Rosario y en las localidades de origen del Chaco, donde todos y cada uno de nuestros interlocutores afirmaron "tener familia" en las periferias de Buenos Aires. Nuestras conversaciones con los jóvenes qom derivaban, tarde o temprano, en anécdotas que enlazaban lugares de origen y lugares de migración, uniendo el monte con la ciudad, haciendo explícito un sentido de pertenencia socio territorial que excede lo local. Entre las nuevas generaciones, la migración, no sólo está presente en las nostálgicas historias y memorias transmitidas por padres y abuelos, sino también en las vivencias cotidianas de quienes tienen "familia" aquí y allá, y que por eso mismo pueden ir y volver, quedarse o regresar. Durante el trabajo de campo, a menudo, le preguntábamos a la gente por las causas de la migración y la mayoría nos hablaba no sólo de las transformaciones que habían sufrido en relación a las condiciones materiales de existencia sino que además enfatizaban la voluntad de estar junto a "los parientes". A partir de estas observaciones analizamos las implicancias del parentesco en la migración y la consecuente redimensionalización territorial ${ }^{24}$, las formas en que los flujos migratorios se encauzan, impulsados por este tipo de vínculos de parentesco, que se trasponen en patrones de residencia de los migrantes constituyendo un campo migratorio que precede y configura sus disposiciones, expectativas y acciones ${ }^{25}$.

En cuanto al tercer aspecto, los migrantes indígenas se encontraron con la imposibilidad de insertarse plenamente en el mercado de trabajo, por lo cual pasaron a desempeñar tareas ocasionales en el sector de la construcción, a realizar servicios domésticos y continuaron con tareas de tipo tradicional como la confección de artesanías. Como consecuencia de esta inserción en el mercado laboral se nuclearon en asentamientos de carácter informal ${ }^{26}$.

\footnotetext{
${ }^{24}$ MAIDANA, Volver a la tierra..., op. cit.

25 IDEM, Migrantes toba..., op. cit.

${ }^{26} \mathrm{Si}$ bien el nucleamiento de referencia no sería en principio de carácter informal dado que, como mencionábamos anteriormente, fue promovido por un plan del Gobierno Provincial, ante la imposibilidad de formalizar la ocupación territorial en términos jurídicos mediante un pago
} 
El análisis de las experiencias concretas y de las subjetividades que se articulan con los elementos estructurales en juego, combinado con una perspectiva de redes sociales nos permitió aprehender, la articulación entre las macro estructuras y la acción individual y colectiva, es decir, entre las políticas sociales e indigenistas llevadas adelante por el gobierno y el esfuerzo de las organizaciones de migrantes; y contextualizar estos procesos a la luz de los cambios políticos y socioeconómicos desarrollados a partir de la conquista y colonización del Gran $\mathrm{Chaco}^{27}$.

Pasaremos ahora a señalar por qué el análisis de redes sociales (ARS) -mediante el uso de programas informáticos (UCINET-NETDRAW)- aplicado a los procesos migratorios, puede constituir una importante contribución en el análisis de las relaciones que estructuran el campo migratorio, posibilitando el acceso al suelo urbano y la reconstrucción de la identidad étnica en el nuevo medio. Reflexionaremos entonces sobre las posibilidades de visualizar y graficar las redes sociales, complementando el análisis etnográfico, para profundizar en el conocimiento de estos complejos procesos, que atañen individuos, grupos e instituciones, sus prácticas y sus relaciones, en un momento dado de su existencia.

\section{El ARS, la migración indígena y la redimensionalización territorial}

El desarrollo de la técnica de redes sociales surgió del análisis de la "adaptación" de migrantes africanos a las ciudades ${ }^{28}$. El concepto de red social (social network) ganó aceptación dentro de las ciencias sociales a partir del trabajo pionero de J. A. Barnes ${ }^{29}$, quien definió la red social como la red de relaciones efectivas o potenciales conectando distintas personas, una vez que las relaciones estructurales en términos de categorías sociales o del sistema de roles han sido abstraídas.

Se considera que las redes sociales juegan un rol crítico en la decisión de emigrar, al reducir el costo de la migración y aumentar la probabilidad de encontrar empleo en el área de destino ${ }^{30}$. Es en este contexto que

\footnotetext{
estipulado, hoy sus habitantes sólo poseen un título provisional de las tierras sobre las cuales autoconstruyeron sus viviendas.

27 MAIDANA, Migrantes toba..., op. cit.

${ }^{28}$ HANNERZ, Ulf. Exploring the City. Toward an Urban Anthropology.

${ }^{29}$ BARNES, John Arundel. Class and comités in a Norwegian Island Parish.

${ }^{30}$ Para el caso de México Skoufias et alii (SKOUFIAS, Emmanuel; Trine LUNDE; PATRINOS, Harry Anthony. Social networks among indigenous peoples in Mexico) señalan la gran cantidad de evidencia empírica existente sobre la significación de las redes sociales en la migración indígena de México a Estados Unidos; remitiendo como ejemplo de ello a los trabajos de Massey y Espinosa (MASSEY, Douglas S.; ESPINOSA Kristin E. What's Driving Mexico-U.S. Migration? A Theoretical, Empirical, and Policy Analysis. American Journal of Sociology, v. 102, n. 4, 1995, p. 939-999.),
} 
proponemos pensar los procesos migratorios del Pueblo Qom, analizados desde 1986 ${ }^{31}$, utilizando los conceptos de flujo de relaciones y redes. Como flujos al interior de territorios/redes que, enlazando actores sociales y lugares, desempeñan un importante papel la estructuración/organización territorial ${ }^{32}$. Reconocemos que esta metodología si bien no alcanza a dimensionar los efectos de la compleja interacción social, enmarcada en el contexto de la etnografía contribuye al estudio de los efectos de las redes no sólo para la migración, sino también para la consecuente redimensionalización territorial operada tras la misma. En líneas generales podemos decir que el ARS, por las bondades que presenta en el tratamiento que involucran aspectos relacionales, es importante para el tratamiento de las migraciones aprehendidas como procesos en los cuales las unidades que los constituyen no están actuando de manera independientemente, sino influenciándose mutuamente. De hecho, como señalamos anteriormente, el análisis de redes sociales en antropología comienza su desarrollo con el estudio de las corrientes migratorias.

Al concebir las redes como mecanismos de movilización de capital social, es decir, de recursos reales o potenciales ligados a la pertenencia a una red duradera de relaciones, de conocimiento y reconocimiento mutuo ${ }^{33}$ y recatando el baje teórico-conceptual de las ciencias sociales latinoamericanas generado en torno al intercambio, específicamente en las migraciones internas del campo a la ciudad ${ }^{34}$, la reciprocidad puede ser vista como el principio organizador de las redes de los migrantes indígenas qom a las ciudades, redes fundadas en el parentesco práctico. Planteamos por ello el estudio de las relaciones/vínculos de parentesco de un conjunto de indígenas qom que habitan uno de los tantos asentamientos que los mismos han constituido -producto de la migración- en diversas localidades de la provincia de Buenos Aires.

El grafo o gráfico bosqueja visualmente la red social considerada [Imagen II]. El mismo está constituido por un conjunto de nodos (puntos) que repre-

\footnotetext{
Orrenius (ORRENIUS, Pia M. The Role of Family Network, Coytotes Prices and the Rural Economy in Migration from Western Mexico: 1965-1994. Working Papers 99-10, Federal Reserve Bank of Dallas, 1999) y Munshi (MUNSHI, Kaivan. Networks In The Modern Economy: Mexican Migrants in The U.S. Labor Market. The Quarterly Journal of Economics, v. 118, n. 2, 2003, p. 549-599). A la vez que ponen de relieve la escasa evidencia sobre el rol de las redes sociales en la migración interna. Temática que abordan en su trabajo confirmando la significación de las mismas en estos tipos de desplazamientos. En el caso de Brasil el trabajo de Figoli y Fazito (op. cit.) discute algunos aspectos de método en el mapeo de redes sociales con migrantes indígenas.

31 TAMAGNO, NAM QOM..., op. cit

${ }^{32}$ BLANCO, Jorge. La geografía de las redes.

${ }^{33}$ BOURDIEU, op. cit.

${ }^{34}$ LOMNITZ, Larissa. Cómo sobreviven los marginados.
} 
sentan a los actores, es decir, a las entidades sociales consideradas. En este caso se trata de individuos, específicamente de referentes ${ }^{35}$ qom que habitan el nucleamiento antes descrito, correspondiendo los triángulos a hombres y los círculos a mujeres. Las relaciones consideradas sobre el conjunto de actores corresponden a relaciones de parentesco; las mismas son de carácter binario, es decir están presentes o ausentes (se es pariente o no se es) y recíprocas (si A1 es pariente de A2, A2 es pariente de A1), de allí que se representen mediante flechas dobles marcando esta relación de ida y vuelta ${ }^{36}$.

\section{IMAGEN II}

Red conformada por referentes indígenas (qom) migrantes nucleados en torno a la Asociación Civil Ntaunaq Nam Qom y sus vínculos de parentesco

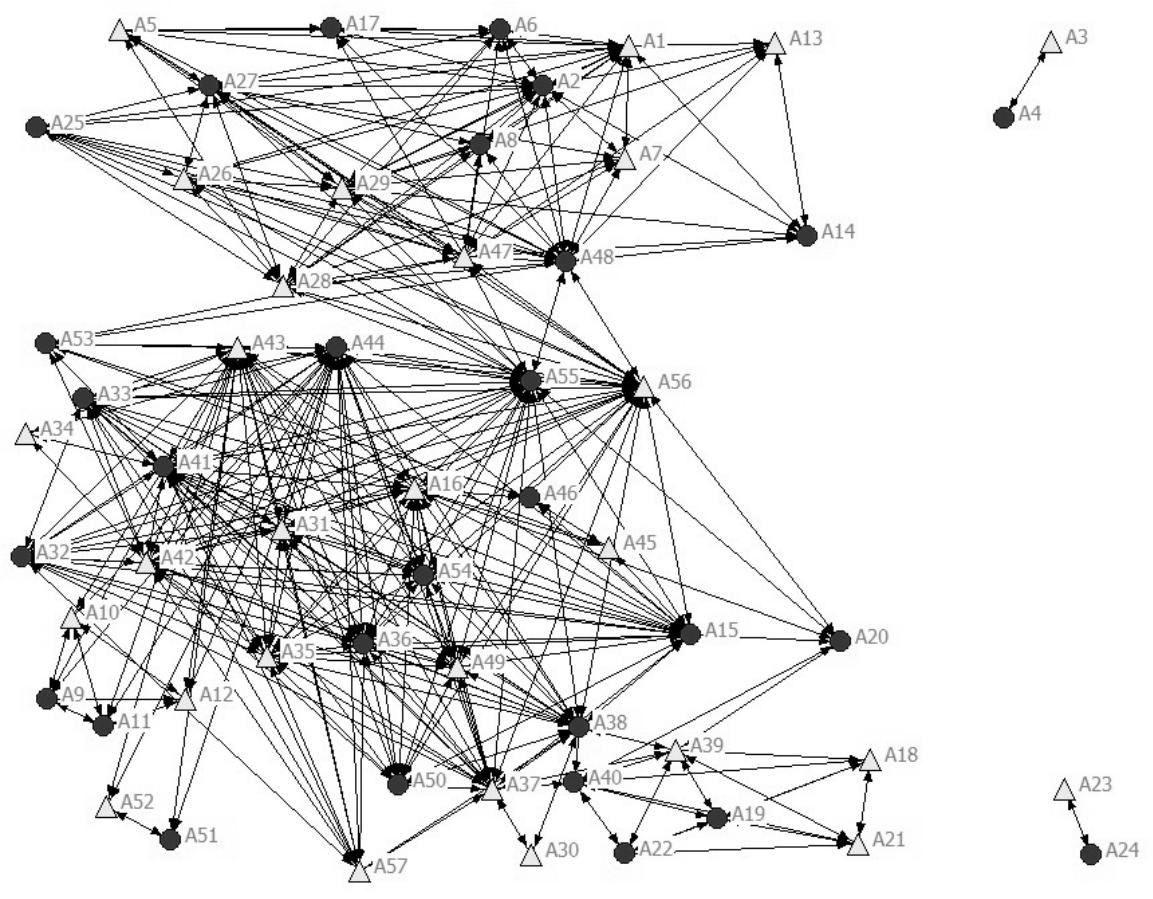

Con la mera observación del grafo correspondiente a la red presentada notamos que la misma posee tres componentes. En base al trabajo etnográfico realizado sabemos que A3-A4 y A23-A24 están unidos entre sí por una relación de alianza, que ambas parejas provienen de Pampa del Indio

\footnotetext{
${ }^{35}$ Se han considerado como referentes a parejas o individuos fundadores de cada una de las 36 unidades domésticas que conforman el nucleamiento de referencia.

${ }^{36}$ La red presentada corresponde a la visualización gráfica mediante NETDRAW de los datos analizados con el programa de Análisis de Redes Sociales UCINET.
} 
-zona de procedencia de la mayor parte de los individuos considerados-, y se vinculan con A35 no sólo por su procedencia sino por ser practicantes del mismo culto. Esto es de suma importancia al momento de considerar la "accesibilidad", dado que prácticamente todos los actores pueden acceder al resto de la red, aunque no presenten vínculos directos con cada uno de los nodos que la conforman, es decir, aunque las distancias entre ellos sean distintas (presenten diferentes longitudes). El concepto de cadena se refiere a la transferencia de apoyo material e información que familiares, amigos y/o paisanos (en el sentido de coresidentes) ofrecen a los potenciales migrantes para decidir y/o concretar su desplazamiento ${ }^{37}$. La información resulta el elemento central de las relaciones reticulares entre individuos. En este sentido son las relaciones genealógicas junto a las relaciones de amistad y paisanaje más cercanas las que parecen representar las vías de comunicación e interacción principales; constituyendo también el culto evangélico (principalmente la Iglesia Evangélica Unida o Iglesia Toba), un sistema de comunicación eficaz, al promover el encuentro entre individuos. Asimismo, reparando en el número de actores y la cantidad de lazos presentes en relación a los posibles, anticipamos una densidad alta. Algo que confirmamos a través del cálculo con UCINET. Pero, si bien la mayoría de los nodos se relacionan entre sí, al observar el grafo resaltan ciertas regiones de la red por su mayor o menor conectividad. Las diferencias en cómo los individuos están conectados puede ser extremadamente útil para entender sus atributos y comportamientos, su significación dentro de la red ${ }^{38}$.

La perspectiva de redes sugiere que el poder de cada uno de los actores surge de sus relaciones con los otros, por lo cual se deduce de la posición ocupada en la red de relaciones. Presentamos aquí una primera aproximación a la estructura de esta red mediante el análisis de tres medidas de centralidad ${ }^{39}$ : rango (degree), grado de intermediación (betweenness) y cercanía (closeness).

El rango (degree) es el número de lazos directos de un actor o nodo, es decir, con cuántos otros nodos se encuentra directamente conectado. En este caso observamos que A43 y A44 tienen los mayores rangos. Lo cual nos indica que son las personas más conectadas de este grupo, las de mayor centralidad. Podríamos suponer que son quienes controlan la mayor cantidad de información. Ya que el rango puede ser considerado una medida

\footnotetext{
37 Mc DONALD, Mc DONALD, op. cit.

${ }^{38}$ HANNEMAN, Robert. Introducción a los métodos del análisis de redes sociales.

39 Por medida de centralidad se entiende un conjunto de algoritmos calculado sobre cada red, que permite conocer la posición de los nodos al interior de la misma y la estructura de la propia red.
} 
que permite acceder al índice de accesibilidad a la información que circula por la red. Siendo los actores de rango más alto los que cuentan con mayor probabilidades de conocer la información y difundirla. El grado también puede ser interpretado como el grado de oportunidad de influir o ser influido por otras personas en la red. La aplicación del programa suministra estadísticos descriptivos, que brindan información sobre los valores que tomó el rango en el conjunto de la red. En este caso el rango promedio de la red (mean) es de 11.123 y los valores oscilan entre 1 y 26 (la menor y la mayor cantidad de lazos contabilizadas entre nodos). Esta medida entonces no sólo nos ofrece una primera aproximación para determinar cuáles son los nodos centrales, sino que al mostrar una alta tasa de conexiones puede estar indicando una significativa capacidad para movilizar recursos y resolver problemas, algo de suma importancia para el caso, dado que nos estamos refiriendo a población migrante. Además, la presencia vínculos parentales directos entre la mayoría de los actores considerados permite dimensionar la importancia del parentesco no sólo durante el proceso migratorio, sino también durante los posteriores procesos de asentamiento y reorganización en los territorios de migración.

El grado de intermediación (betweenness) indica la frecuencia con que aparece un nodo en el tramo más corto (o geodésico) que conecta a otros dos. Es decir, muestra cuando una persona es intermediaria entre otras dos del mismo grupo, cuando actúa de "puente". En este caso A 55 y A56 son las personas con mayor grado de intermediación. Si observamos el gráfico vemos que conectan dos grandes bloques dentro del mismo. De acuerdo a la información recabada durante el trabajo de campo sabemos que estos bloques expresan diferentes líneas de descendencia. Le siguen A43 y A44 que también actúan como "puente" entre dichos bloques.

El grado de cercanía (closenness) indica la cercanía de un nodo respecto al resto de la red, representa su capacidad de alcanzar a los demás. A56 y A55 son quienes cuentan con un grado de cercanía más grande. A ellos les siguen A44 y A43. Estos últimos son los mismos que aparecen como centrales al considerar la medida de grado. Es interesante notar que los mayores niveles de Intermediación (betweenness), si bien se corresponden a grandes rasgos con los de grado y cercanía, muestran a A56 y A55 como los actores de mayor relevancia en cuanto al acceso total a la red, pues son ellos quienes presentan los valores más altos. Esto podría relacionarse con el denominado "efecto llamada", el hecho de ser capaz de "alcanzar" al resto de la población con menos esfuerzo que los demás integrantes de la red estaría señalando a estos cuatro nodos como eslabones centrales de la 
cadena migratoria, como personas con un lugar privilegiado para informar sobre las condiciones en el destino de migración a quienes habitan los lugares de origen, para animarlos a emigrar, para hospedarlos a su llegada, para ayudarlos a asentarse y a organizarse en el nuevo medio.

En términos cualitativos este análisis implica la posibilidad de valorar la significación de determinados nodos que aparecen ocupando posiciones centrales. La ventaja radica en que a través de este tipo de estudios se puede establecer la complejidad y aprehender la dinámica de las cadenas y redes migratorias no sólo a través de los testimonios de cada uno de los protagonistas, sino también, mostrando la importancia de las relaciones establecidas entre ellos, de su posición en la red. La información etnográfica indica que los nodos que aparecen ocupando lugares centrales en la red considerada, corresponden a parejas que unidas por alianza vinculan a la familia del primer presidente de la asociación y del actual "cacique" con otras líneas de descendencia presentes en el barrio. En esta red, altamente conectada, las posiciones estratégicas asumidas por individuos que ocupan la intersección de las relaciones los convierten en actores principales de los procesos de territorialización; pues les permite movilizar distintos tipos de capital necesarios para la migración, acceso al suelo urbano y organización socio-territorial. En este sentido se cumple el hecho de que las posiciones anteriormente descriptas posibilita la eficaz activación de una porción de la red total con fines específicos, lo que Mayer ${ }^{40}$ denomina "action-set".

Las relaciones parentales del migrante actúan como fuerza que los hace no sólo regresar periódicamente a los lugares de origen y/o mantener vínculos de distinta índole con ellos; sino también nuclearse en los lugares de migración. "Primero llegué a Quilmes y entonces busqué a mi mamá en La Plata. No sé si son los toba o los criollos son igual... desde que conocí a mi mamá, la encontré de grande ya, siempre quise vivir cerca de ella" (Mujer indígena qom, ciudad de La Plata, año 2010)

\section{Consideraciones finales}

Los conceptos de cadena migratoria y red así como el ARS posibilitan comprender los movimientos poblacionales indígenas y la consecuente construcción territorial como procesos continuos y dinámicos de aceptación/ rechazo a las presiones recibidas desde lo hegemónico; y a la constitución de "barrios toba" en los lugares de destino de sus migraciones, en términos de transposición, reproducción y recreación de redes sociales en particulares contextos políticos, económicos y socioterritoriales. En este sentido afirmamos

${ }^{40}$ MAYER, Adrian. The Significance of Quasi-Groups in the Study of Complex Societies. 
que la aplicación de estas propuestas teóricas-metodológicas a lo largo del trabajo de campo nos ha permitido corroborar la forma en que las mismas enriquecen distintos niveles de análisis de la investigación.

La observación de la vigencia de los lazos de parentesco establecidos en y con los lugares de origen, nos permitió afirmar que éstos contribuyen a mantener viva la memoria genealógica, permitiendo que los migrantes se reconozcan en un origen común y en una pertenencia, y que se identifiquen como parte de un Pueblo más allá de su situación geográfica. El análisis de los gráficos de las relaciones parentales de los migrantes nos permitió no sólo reafirmar que son este tipo de vínculos los que actúan como fuerza que los hace regresar periódicamente a los lugares de origen y/o mantener vínculos de distinta índole con ellos, sino también nuclearse en los lugares de migración.

Si bien reconocemos que esta metodología no alcanza a dimensionar los efectos de la compleja interacción social, enmarcada en el contexto de la etnografía contribuye al estudio de los efectos de las redes no sólo para la migración, sino también para la consecuente redimensionalización territorial operada tras la misma.

Las nuevas tecnologías informatizadas para el análisis de redes sociales (ARS) constituyen herramientas que permiten no sólo una mejor visualización gráfica de las redes sociales, sino y al mismo tiempo expresarlas en su densidad relativa, mostrando determinadas características como ser, en este caso, la significación de ciertos individuos en la constitución de las mismas, en la generación de los movimientos migratorios y en las concentraciones poblacionales en los lugares de migración.

En este sentido, el trazado de las redes de parentesco, a partir de la investigación etnográfica realizada, fue una herramienta teórica-metodológica crucial para el análisis, al permitir no sólo la identificación de individuos clave en los procesos de migración e instalación en el medio urbano, sino por constituir una fuerte evidencia de la cohesión social que logran los qom migrantes en algunos espacios, más allá de la dispersión/discontinuidad física y de las posibles tensiones existentes, propias de toda relación social.

\section{Bibliografía}

ARANGO, Joaquín. Las 'leyes de las migraciones' de E. Ravenstein, cien años después. Revista Española de Investigaciones Sociológicas, v. 32, 1985, p. 7- 26.

ARIAS, Nora Julia. Vueltas y revueltas (estratégicas) de los Toba rosarinos - de Resistencia a Rosario. Tesis doctoral. Rio de Janeiro: PPGAS/MN/Universidade Federal de Rio de Janeiro, 2005.

ARIZPE, Lourdes. Migración indígena, problemas analíticos. Nueva Antropología, n. 
5, 1976, p. 63-89.

ARIZPE, Lourdes. Migración, etnicismo y cambio económico (un estudio sobre migrantes campesinos a la ciudad de México). México: El Colegio de México, 1978.

BARNES, John Arundel Class and comités in a Norwegian Island Parish. Human Relations, v. 7, 1954, p. 39-58.

BARTOLOMÉ, Miguel. Los pobladores del desierto. Genocidio, etnocidio y etnogénesis en la Argentina. Amérique Latine Histoire et Mémoire. Les Cahhiers ALHIM, v. 10, 2004. Disponible en: <http://alhim.revues.org/index103.html>.

BASSARSKY, Lina. Enfoque de redes sociales en las migraciones de América latina hacia Francia. Anuario de Estudios Americanos, v. 64, n., 2007, p. 141-172.

BIGOT, Margot; Graciela RODRÍGUEZ; Héctor VÁSQUEZ. Asentamientos TobaQom en la ciudad de Rosario. Procesos étnicos identitarios. América Indígena, v. 51, n. 1, 1991, p. 217-251.

BLANCO, Jorge. La geografía de las redes. In FERNÁNDEZ CASO, M. Victoria (coord.). Geografía y territorios en transformación. Nuevos temas para pensar la enseñanza. Buenos Aires: Noveduc, 2007, p. 39-66.

BOURDIEU, Pierre. El sentido práctico. Madrid: Taurus Humanidades, 1991.

EISENSTADT, Shmuel N. The absorption of Inmigrants. Londres: Routledge \& Kegan Paul, 1954.

FÍGOLI, Leonardo; FAZITO, Dimitri. Redes sociales en una investigación de migración indígena: el caso de Manaus. Revista Brasileira de Estudos de População, v. 26, n.1, 2009, p. 77-95.

HANNEMAN, Robert. Introducción a los métodos del análisis de redes sociales. California: Departamento de Sociología de la Universidad de California Riverside, 2000.

HANNERZ, UIf. Exploring the City. Toward an Urban Anthropology. New York: Columbia University Press, 1980.

LOMNITZ, Larissa. Cómo sobreviven los marginados. México: Siglo XXI, 1976.

MAIDANA, Carolina Andrea. VOLVER A LA TIERRA. Parentesco, redimensionalización territorial y reconstrucción identitaria. TAMAGNO, Liliana (org.). Pueblos Indígenas. Interculturalidad, colonialidad, política. Buenos Aires: Biblos, 2009, p. 45-57.

. Migrantes toba (qom). Procesos de territorialización y construcción de identidades. Tesis Doctoral. La Plata: Universidad Nacional de La Plata, 2011.

MARGULLIS, Mario. Migración y marginalidad en la sociedad argentina. Buenos Aires: PAIDOS, 1968.

MAYER, Adrian. The Significance of Quasi-Groups in the Study of Complex Societies. In BANTON, Michael (ed.). The Social Anthropology of Complex Societies. London: Tavistock, ASA, 1966, p. 97-122.

MC DONALD, Leatrice; MC DONALD, John Stuart. Chain migration, ethnic neighborhood Formation and social networks. The Milbank Memorial Fund Quaterly, v. XLII, n. 1, 1964.

RAVENSTEIN, Ernest George. The Laws of Migration. Journal of the Royal Statistical Society, v. 48, n. 2, 1885, p. 167- 235. 
. The Laws of Migration. Journal of the Royal Statistical Society, v. 52, n. 2, 1889, p. 241-305.

RECAÑO VALVERDE, Joaquín. El papel de las redes en los procesos de migración interna. Revista de Demografía Histórica, v. 20, n. 1, 2002, p. 15-20.

SKOUFIAS, Emmanuel; Trine LUNDE; PATRINOS Harry Anthony. Social networks among indigenous peoples in Mexico. Latin American Research Review, v. 45, n. 2, 2010, p. 49-67.

TAMAGNO, Liliana. NAM QOM HUETA'A NA DOQSHI LMA'. Los tobas en la casa del hombre blanco. Identidad, memoria y utopía. La Plata: Editorial Al Margen, 2001. - Identidades, saberes, memoria histórica y prácticas comunitarias. Indígenas tobas migrantes en la ciudad de La Plata, capital de la Pcia. de Buenos Aires Argentina. Revista CAMPOS, edición especial, 2003, p. 165-182.

TAMAGNO, Liliana; GARCÍA, Stella Maris; IBÁÑEZ CASELLI, María Amalia; GARCÍA, María del Carmen; MAIDANA, Carolina; ALANIZ, Marcela; SOLARI PAZ, Verónica. Testigos y protagonistas: un proceso de construcción de conocimiento conjunto con vecinos QOM. Una forma de hacer investigación y extensión universitaria. Revista Argentina de Sociología, año 3, n. 5, 2005, p. 206-222.

WRIGHT, Pablo. Histories of Buenos Aires. In MILLER, Elmer (ed.). Peoples of Gran Chaco. London: Bergin and Garvey, 1999, p. 35-156.

\section{Abstract}

\section{Indigenous migration, territorialization processes, and social network analysis}

This paper discusses the scopes and limitations of social network analysis in the study of indigenous migration and consequent territorialization processes. From the data obtained in a study on the formation of called "toba neighborhoods" in the peripheries of large cities, we describe and analyze the social network that connects, through kinship, the inhabitants of one of these neighborhoods. Finally we describe the relationship between the centrality of each individual in the network architecture and its contribution to explain the direction of migration, and the subsequent settlement in a particular area.

Keywords: indigenous, social networks, kinship, migration, processes of territorialization.

Recebido para publicación en 31/08/2012.

Aceptado para publicación en 19/03/2013.

Received for publication in August, 31 ${ }^{\text {th }}, 2012$.

Accepted for publication in March, 19 ${ }^{\text {th }}, 2013$.

ISSN impresso: 1980-8585

ISSN eletrônico: 2237-9843 